\title{
Research on the Influence of Smartphone Navigation on Driving Behavior Based on Real Vehicle Driving
}

\author{
Chen-hao Dong $\mathbb{D}^{1,2}$ Rong-guo Ma, ${ }^{1}$ Dong Zhang $\mathbb{D}^{1},{ }^{1}$ Wan-ting Zhang, \\ and Fang-fang Wang ${ }^{1}$ \\ ${ }^{1}$ Department of Traffic Engineering, School of Highway, Chang'an University, Xi'an 710064, China \\ ${ }^{2}$ Department of Xi'an City Planning \& Design Institute, Xi'an 710064, China \\ Correspondence should be addressed to Dong Zhang; 294856372@qq.com
}

Received 29 December 2018; Revised 8 May 2019; Accepted 3 June 2019; Published 13 June 2019

Academic Editor: Juraj Machaj

Copyright (c) 2019 Chen-hao Dong et al. This is an open access article distributed under the Creative Commons Attribution License, which permits unrestricted use, distribution, and reproduction in any medium, provided the original work is properly cited.

\begin{abstract}
This paper discusses a study of the influence of different positions and modes of smartphone navigation devices on driving behavior based on real vehicle driving. Twenty professional drivers participated in the experiment in free-flowing traffic and good weather conditions. The eye movement and vehicle control data obtained in the experiment were used as indicators for evaluation. Comparison of the mean, variance, and significance analysis shows that a smartphone navigation device placed on the right side of the car dashboard (position 1) has less impact on driving behavior than when placed above the air conditioning vent (position 2). A smaller angle of view can increase the fixation frequency and the length of time that the driver spends looking out the windshield and reduce the range and time spent in glancing at the navigation device. Using only sound navigation (the audio group in this paper) has the least influence on driving behavior because the driver's visual attention is not transferred inside the vehicle rather than on the road ahead, and the vehicle is operated in the most stable state. These findings have practical significance for reducing the negative influence of smartphone navigation.
\end{abstract}

\section{Introduction}

Navigation systems can provide drivers with real-time, accurate road condition information and travel routes, which are especially important in unfamiliar road conditions and in a complex road network [1]. In the early days, in-vehicle navigation was the main navigation mode $[2,3]$. However, with the rapid development of science and technology, smartphone navigation has gradually replaced traditional invehicle navigation [4]. In terms of driving performance and vehicle control, smartphone navigation is superior to invehicle navigation on urban and suburban roads, and it is more fuel-efficient in unfamiliar traffic conditions $[5,6]$. The navigation system brings many benefits to the driver, but public attention focuses on its efficiency and convenience, while ignoring the negative effects. Multiple tasks need to be performed simultaneously during driving, including cognition, perception, and movement. Navigation systems usually involve vision, operation, and cognition, which are the same tasks that are needed for driving, and cause significant interference [7]. Therefore, exploring the influence of navigation systems on driving behavior has become an important research field.

During the driving process, the use of smartphone navigation leads to the generation of secondary tasks, such as inputting the destination, viewing the navigation route, and listening to vocal instructions. In addition, the driver's reaction time to a traffic incident is slower due to distracted behavior, which causes more emergency braking [8]. The ISO (International Organization for Standardization) indicates that a variety of vehicle information systems, such as mobile phones, navigation systems, and radios, can distract drivers and create traffic hazards [9]. A German study classified and evaluated the types of driving and secondary tasks. The results showed that secondary tasks had negative effects on driving behavior [10]. Moreover, the workload 
generated by the driving task also varies when the driver operates the navigation system [11]. Many researchers have carried out further research through specific experiments. Based on the data analysis of reaction time, vehicle position, speed, and driving distance, White et al. [12] found that the use of mobile phones in driving seriously interfered with the performance of driving tasks. A phone conversation while driving has an impact on cognition and thus reduces driving effectiveness. A study showed that test vehicles had shorter space headway, higher accident rates, and longer brake response time [13]. Different musical rhythms can also affect the driver. The faster the music tempo, the faster the simulated driving speed, and drivers often ignore a red light and drive through zebra crossings [14].

The most important sensory organ in driving is vision: $90 \%$ of driving information is transmitted to the driver through vision [15]. Therefore, many studies take eye movement as the evaluation indicator. Drivers carried out various secondary in-vehicle tasks while driving, and a HED (head-mounted eye-tracking device) was used to collect drivers' fixation indicators and pupil diameter data. The results showed that the standard fixation deviation is shorter than is observed in normal driving in the task of calculating the meeting date [16]. However, using the HED to collect eye movement may make driving behavior unnatural [17]. The appearance of the portable eye tracker solves this problem. In a 2011 experiment, drivers were divided into two groups (group 1 performed a read number task and group 2 performed a select menu system task). The results showed that fixation counts and fixation time in group 1 were smaller than those in group 2 [18]. Japanese researchers used two different in-vehicle navigation screen sizes (one of 7 inches and the other of 4.3 inches) to carry out multiple experiments in different positions. They found that the large screen glance frequency was shorter than the small screen glance frequency, and the large screen glance duration was shorter than the small screen glance duration [19]. A driving simulation experiment was carried out by twenty participants. When the driver's vision and cognition were occupied with navigation information, the driver's operating extensive time and numbers of errors in operation were analyzed [20]. Other studies have shown that drivers' secondary task response to auditory and audiovisual stimuli are faster than their response to purely visual stimuli, and the sound mode helped reduce the interference effects of the secondary task $[21,22]$. Scholars have also studied how to determine the driver's aggressiveness and level of distraction. After analyzing the vehicle trajectory to identify the driver's behavior, the automatic vehicle navigation algorithm (Autono Vi) was optimized and extended to reduce the influence of navigation on the driving behavior [23]. Cheung proposed an autonomous driving planning algorithm that considered the behavior of neighboring drivers' behaviors, which greatly improved the safety and effectiveness of the navigation system [24]. These studies show that people have a deep understanding of the secondary tasks that are generated in the process of driving, but the impact of the smartphone as a navigation tool on driving behavior has not been studied extensively. Smartphone navigation is widely used because it is easy to carry and can be positioned anywhere by the driver. However, different positions and different navigation modes have different effects on driving behavior, so it is necessary to study these variables.

Driving experiments have included simulated driving and real driving, but most of the existing studies are based on driving simulation, which does not reflect authentic data. Real driving experiments can collect highly precise, comprehensive, real data. Consequently, we selected eye movement and vehicle control as the evaluation indicators. We analyzed the influence of smartphones on driving behavior in two different phone positions and three different navigation modes based on real driving and then determined the merits.

\section{Methods}

2.1. Experiment Devices and Indicators. The experiment mainly collected driver eye movement data $[25,26]$ and the vehicle's horizontal and longitudinal operation control. The eye movements were obtained by the portable eye tracker SMI ETG 2w (Berlin, Germany). This device is easy to wear and comfortable and can obtain highly precise data from the driver under relatively natural conditions. The vehicle control data were obtained by a car-following device called Hi-Driver 10 (Beijing, China). This device can collect the absolute speed and traveling speed of the vehicle, acceleration, relative distance between the front and rear car, and following time and calculate the lateral offset. At the same time, the vehicle data recorder that is used in conjunction with the system can collect the video of the vehicle-driving process. GPS records the speed of the vehicle and the driving route at the same time. The user can import GPS data into third-party mapping software, draw the driving route map, and calculate the travel time. It also includes accessories such as smartphones, an automobile data recorder, and a laptop. Specific device functions and required experimental indicators are shown in Table 1.

2.2. Participants and Test Section. Twenty healthy participants with a C1 driver's license (quasi-driving type in China: car) were selected. Participants were 15 males and 5 females, whose details are presented in Table 2. In addition, their experience in using smartphone navigation was 1.6 years. The participants ensured more than eight hours of sleep before the experiment. The experiment also requires two assistants, who were responsible for installation and adjustment of the devices.

The testing area was selected from the Chang'an District (Xi'an, Shaanxi, China); its location and driving route are shown in Figure 1. Traffic conditions and the road alignment of this area are all good. The total length of the test route was $3.4 \mathrm{~km}$, and the specific road attributes are shown in Table 3. The experiment was carried out in good weather conditions. The experiment time was 9:00-11:00 and 14:00-17:00, which avoided rush hours. To obtain better experimental data, we set the two necessary intersections as shown in Figure 1, 
TABLe 1: Devices and indicators

\begin{tabular}{lcccc}
\hline Device name & Number & Function & Classification & Indicator name \\
\hline SMI ETG 2w & 1 & To obtain eye movement indicators & $\begin{array}{c}\text { Fixation } \\
\text { Glance } \\
\text { Blink }\end{array}$ & $\begin{array}{c}\text { Fixation frequency; fixation time } \\
\text { Glance range; glance speed } \\
\text { Blink frequency; blink time }\end{array}$ \\
Hi-Driver10 & 1 & To obtain vehicle data indicators & $\begin{array}{c}\text { Longitudinal } \\
\text { Speed; acceleration; space headway } \\
\text { Lateral offset }\end{array}$ \\
$\begin{array}{l}\text { Automobile data recorder } \\
\text { Smartphone }\end{array}$ & 1 & To record the driving process & - & - \\
Laptop & 2 & Navigation; to record time & - & - \\
Battery & 1 & To collect and save data & - & - \\
\hline
\end{tabular}

TABLE 2: Details of the experiment participants.

\begin{tabular}{lcc}
\hline & Male & Female \\
\hline Frequency & 15 & 5 \\
Max (year) & 38 & 35 \\
Min (year) & 25 & 27 \\
Mean age (year)/standard deviations & $32.3 / 3.9$ & $31.2 / 2.9$ \\
Mean age (year)/standard deviations & \multicolumn{2}{c}{$31.9 / 2.9$} \\
\hline
\end{tabular}

forming a route with several turns, and the driver needed to rely on navigation repeatedly.

2.3. Design and Procedure. Different positions of the smartphone affect the driver's convenience in viewing and obtaining map navigation information. In the driving process, the driver can obtain navigation information from two different information channels: visual and auditory. Therefore, the experiment was conducted with the smartphone position and navigation mode as independent variables. In this paper, two positions were determined based on the size of the smartphone $(14.1 \mathrm{~cm}$ in length and $7 \mathrm{~cm}$ in width), the internal space of the vehicle, and the visual convenience of the driver, as shown in Figure 2. Navigation modes included listening to the navigation voice only, looking at the navigation map only, and an audiovisual combination (the driver chose freely, according to his or her own needs, to listen or watch to get navigation information). In addition, a control group engaged in normal driving without smartphone navigation.

To reduce the data error caused by driving on a familiar route, the experiment first allowed drivers to perform the voice group test with the two placement positions, which prevented the driver from remembering the driving route by watching. Then the audiovisual combination group experiment was carried out. Finally, the visual group experiment was performed. The 20 subjects were involved in experiments under all conditions. That is, each driver drove on the road with two smartphone positions and in 3 navigation modes, respectively. In addition, there was one experimental control group, so there are seven groups of experiments in total.

Between each group of experiments, the participants took an 8-minute break to alleviate driving fatigue. During the experiment, traffic rules were rigorously observed. If major emergencies affected the experiment, such as traffic accidents and bad weather, the experiment was stopped immediately. The specific steps of the experiment were as follows:

Step 1: The devices were installed and debugged. The $\mathrm{Hi}$-Driver was fixed on the hood of the test vehicle, and the direction and angle of the infrared rays were adjusted. The smartphone time was recorded as the standard time, and the difference between the data collection time and the standard time of the instrument was recorded separately. In a later stage, the difference was processed to achieve data synchronization (Figure 3(a)).

Step 2: Fixation points were adjusted and corrected. When participants wore an eye tracker, the experimental assistant ensured that the driver's pupils and iris were directly exposed to the eye tracker's eye movement information acquisition camera. The participant looked at the tip of a fixed object as a reference object. The experimental assistant adjusted the participant's fixation point displayed by the smartphone collector to the fixation point of the reference, and then completed the eye movement calibration (Figures 3(b) and 3(c)).

Step 3: The navigation route was obtained. According to the requirements of the specific experimental group, the experimental assistant placed the smartphone in the specified position and input the origin and destination in the smartphone navigation software to obtain the navigation route.

Step 4: The participant conducted real vehicle driving according to the experimental requirements. During the experiment, the experimental assistant took photos of the driving scene information and recorded the parking time for a long time, which provided a reference for the time synchronization problem in the later data processing. The road traffic environment is shown in Figure 3(d).

2.4. Data Processing. The raw vehicle operation data was derived from the Drive Recorder 3 radar speed measurement software. Begaze software exported all the eye movement data. Therefore, it was necessary to calibrate the inside-vehicle, outside-vehicle, and smartphone fixation data required in the study. The specific calibration process was as follows: The experimenter opened the Begaze software, imported the collected eye movement data packet 


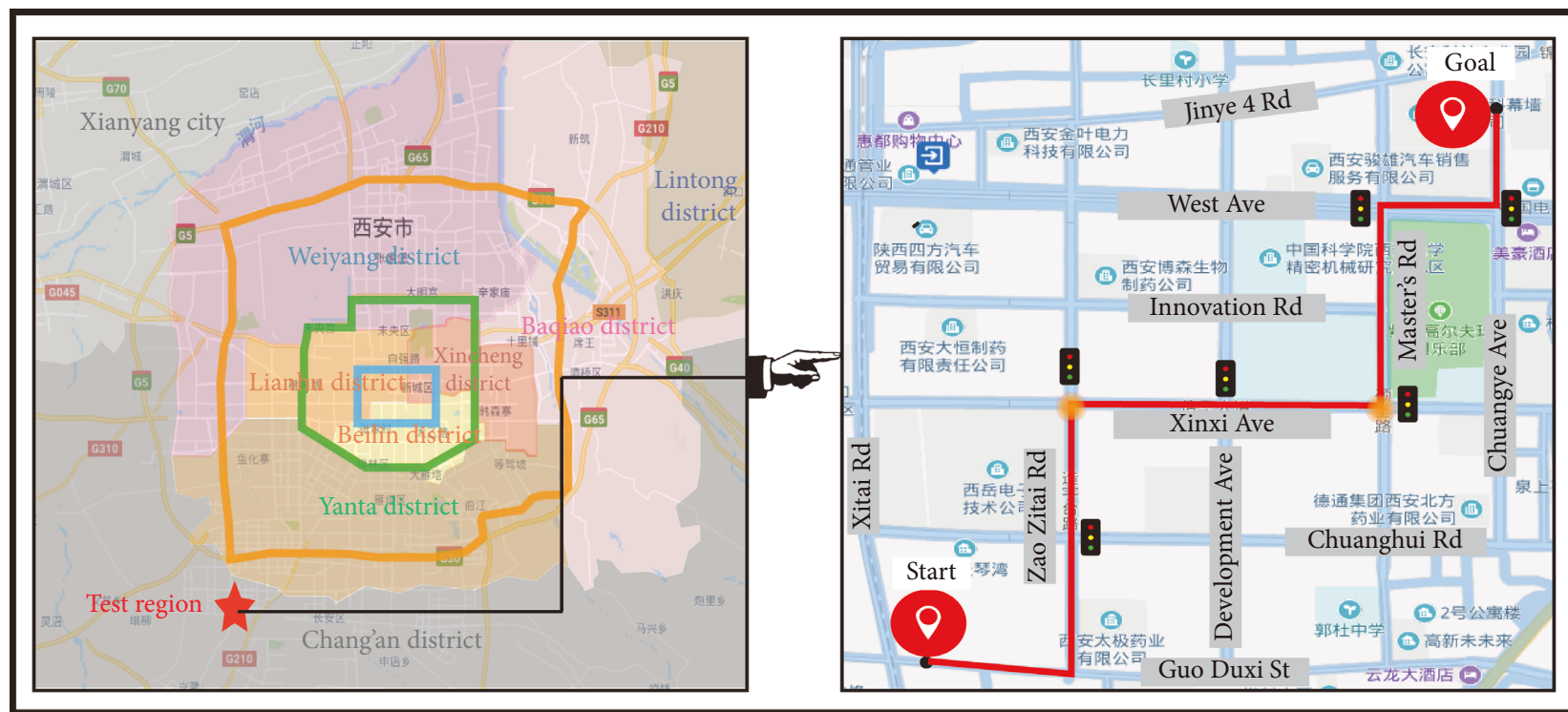

Downtown

Second ring

Third ring

Figure 1: Test roadmap.

TABLe 3: Attributes of each road.

\begin{tabular}{|c|c|c|c|c|c|c|c|}
\hline \multirow{2}{*}{ No. } & \multirow{2}{*}{ Name } & \multirow{2}{*}{ Level } & \multirow{2}{*}{ Number of lanes (two-way) } & \multicolumn{4}{|c|}{ Width $(\mathrm{m})$} \\
\hline & & & & Nonmotor vehicle & Sidewalk & Each lane & Red line \\
\hline 1 & Guo Duxi St & Minor & Two & 1.5 & 1 & 3.5 & 14 \\
\hline 2 & Zao Zitai Rd & Minor & Four & 2 & 3 & 3 & 30 \\
\hline 3 & Xinxi Ave & Major & Six & 3.5 & 2.5 & 3.5 & 40 \\
\hline 4 & Master's Rd & Minor & Four & 2 & 3 & 3 & 30 \\
\hline 5 & West Ave & Major & Six & 4 & 2.5 & 3 & 45 \\
\hline 6 & Chuangye Ave & Major & Six & 3 & 2.5 & 3 & 35 \\
\hline 7 & Xitai Rd & Major & Six & 3.5 & 3 & 3.5 & 45 \\
\hline 8 & Development Ave & Minor & Four & 4 & 2 & 3.5 & 35 \\
\hline 9 & Chuanghui Rd & Minor & Four & 1.5 & 3 & 3 & 26 \\
\hline 10 & Innovation $\mathrm{Rd}$ & Minor & Four & 2 & 3 & 3 & 30 \\
\hline 11 & Jinye 4 Rd & Branch & Two & 1 & 3 & 3 & 25 \\
\hline
\end{tabular}

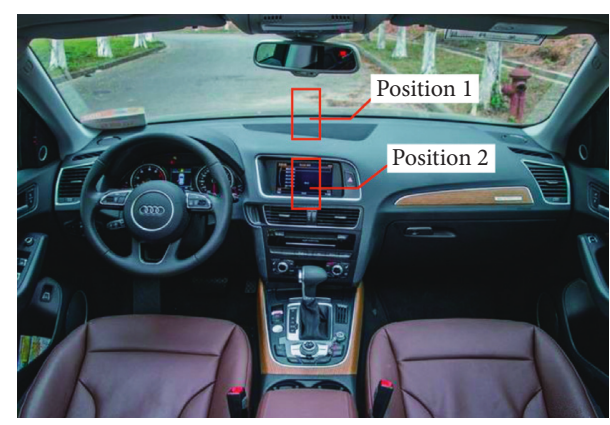

Figure 2: Positions of the smartphone.

into the software, and selected the data area to be obtained. Taking smartphone eye movement data as an example, in the AOI editing, the smartphone area is manually selected. Figure 4 shows that the orange encircled area is the calibrated smartphone area. The calibration process is simple, but the calibration workload is heavy. In addition, due to its acquisition of data in accordance with each frame, the difference between adjacent data is only $42 \mathrm{~ms}$, and the adjacent five consecutive data are basically the same, which cannot reflect the change rule of the data, and errors will occur in data processing. Therefore, a set of data was taken every 0.5 seconds on the basis of the original data for analysis.

A small part of the data obtained by the portable eye tracker and the car-following device had some errors such as attribute defect, data duplication, and abnormal data. There were also emergencies and abnormal vehicle operation during the experiment. Therefore, these data were removed before the data were analyzed. The Pauta criterion is widely used because of its convenience and simplicity, and it is suitable for large sample data. Therefore, this criterion was 


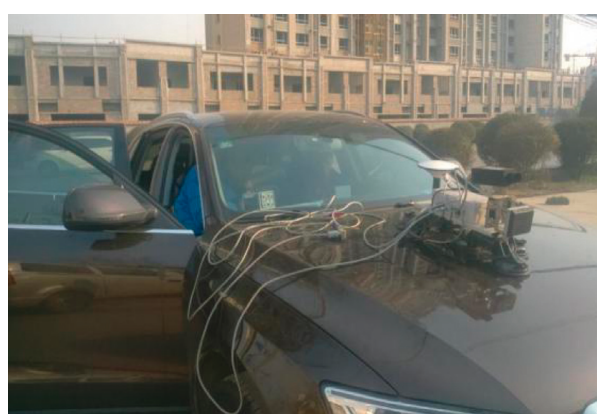

(a)

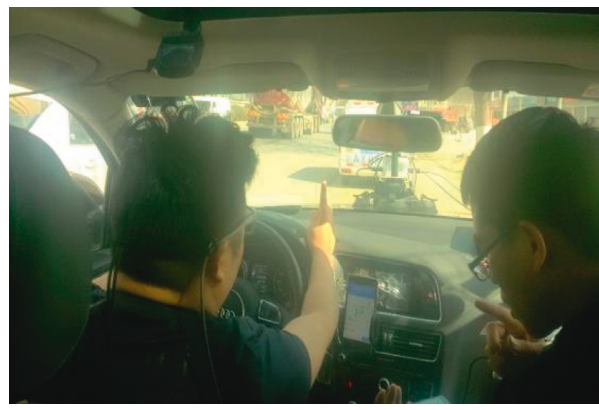

(c)

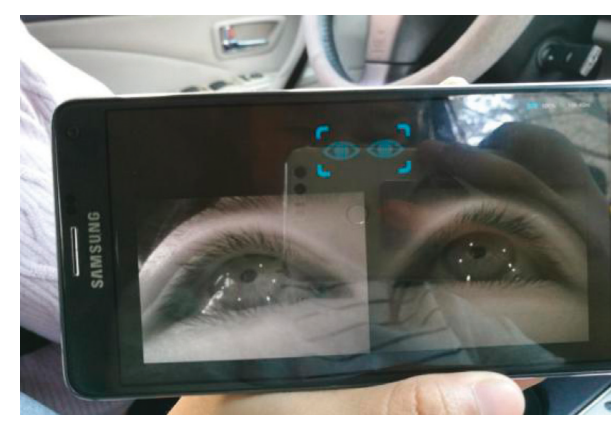

(b)

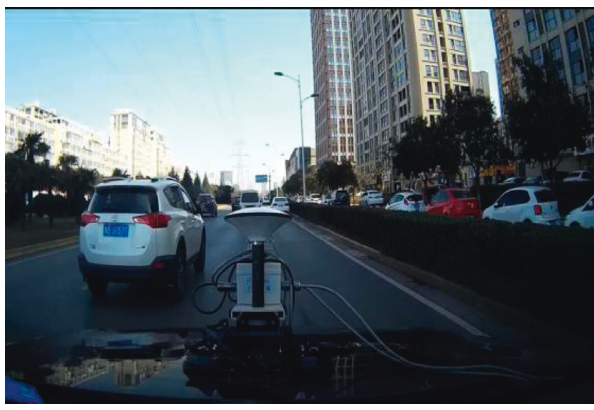

(d)

Figure 3: Steps of real vehicle driving. (a) Fix test equipment, (b) eye movement calibration interface, (c) calibration of eye movement, and (d) road traffic environment.

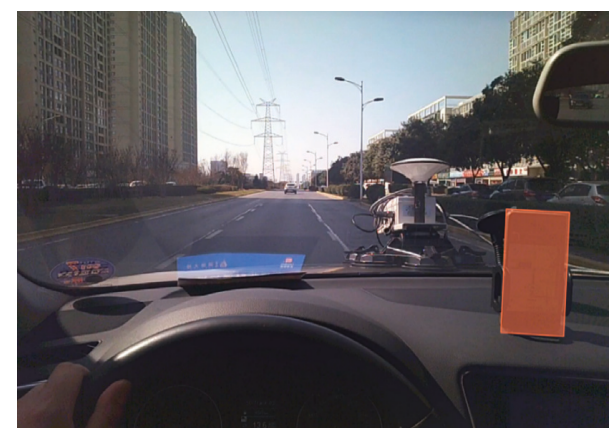

FIGURE 4: Calibration of the smartphone area.

utilized to eliminate the eye movement and vehicle control indicator outliers.

When the difference between the measurement data and the arithmetic mean of the total measurement data was greater than three times the standard deviation, the data were eliminated. The formula is as follows:

$$
\left|x_{i}-\bar{x}\right|>3 \sigma,
$$

where $x_{i}$ is the measurement data, $\bar{x}$ is the arithmetic mean of the total data, and $\sigma$ is the standard deviation.

\section{Results}

In this study, the experimental data were classified according to position and navigation mode and then driving behavior was descriptively analyzed and significant analysis by independent sample $T$ test was performed. (The Kolmogorov-Smirnov test showed that all samples are subject to normal distribution and satisfy the precondition of an independent sample $T$ test).

3.1. The Influence of Different Smartphone Positions. The evaluation indicators and significance $(p=0.05)$ of different smartphone positions are shown in Figures 5 and 6 . The error lines in all the figures in the result are drawn by standard deviation.

The inside fixation frequency $(M=22.66, S=2.96)$ of position 2 is higher than that of position $1(M=19.27$, $S=2.63)$, and both are higher than the control group $(M=12.61, \mathrm{SD}=2.42)$, whereas the outside fixation frequency $(M=83.82, \mathrm{SD}=4.69)$ is less than position 1 $(M=99.56, \mathrm{SD}=4.21)$ and the control group $(M=91.38$, $\mathrm{SD}=4.16)$. In addition, the average inside fixation time of position $2(M=268.33, \mathrm{SD}=11.25)$ is the largest, whereas its average outside fixation time is $29.16 \mathrm{~ms}$ less than position 1 $(M=239.17, \mathrm{SD}=10.32)$ and $31.90 \mathrm{~ms}$ less than the control group $(M=236.43, \mathrm{SD}=10.68)$. This means that when drivers use smartphone navigation, their attention is distracted. By focusing too much on the inside of the car, their attention on the road is reduced. Compared with position 1 , smartphone navigation has a greater effect on the driver at position 2 , and the inside fixation frequency $(t[138]=1.828$, $p=0.029)$ and the average inside fixation time $(t[138]=$ $1.744, p=0.036$ ) have significant effects. Because position 2 is farther away than position 1 from the anterior fixation area of the vehicle, the glance range and speed at position 2 $(M=19.89, \mathrm{SD}=2.71$ and $M=138.25, \mathrm{SD}=12.86)$ are higher than those at position $1(M=11.98, \mathrm{SD}=2.36$ and $M=99.16$, $\mathrm{SD}=11.53)$ when the driver watches the smartphone 


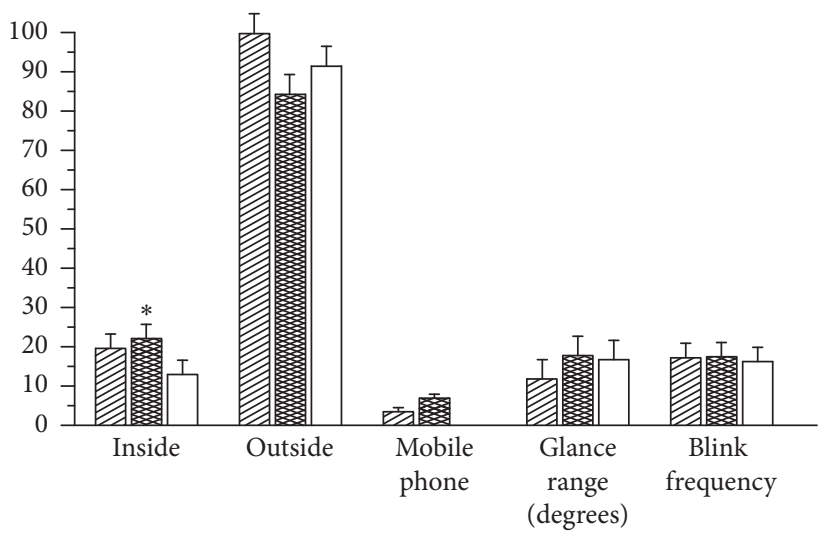

Fixation frequency (three parts)

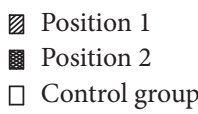

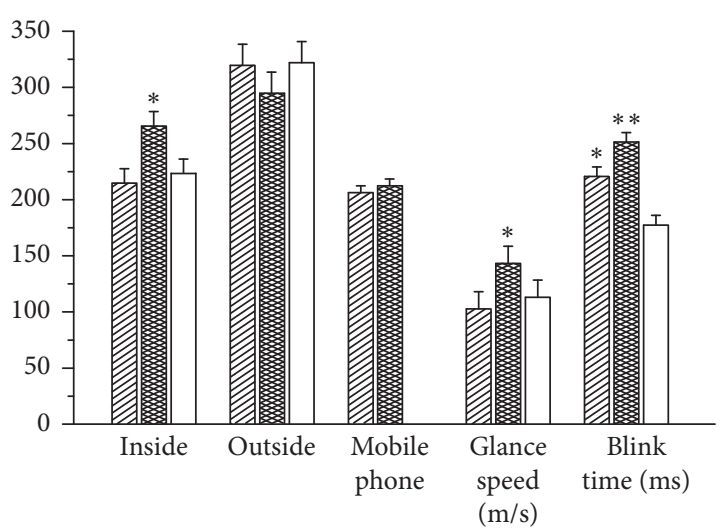

Average fixation time (ms) (three parts)

Position 1

Position 2

$\checkmark$ Control group

(a)

(b)

FIgURE 5: Eye movements for different smartphone positions. (a) Eye movements (part 1); (b) eye movements (part 2).

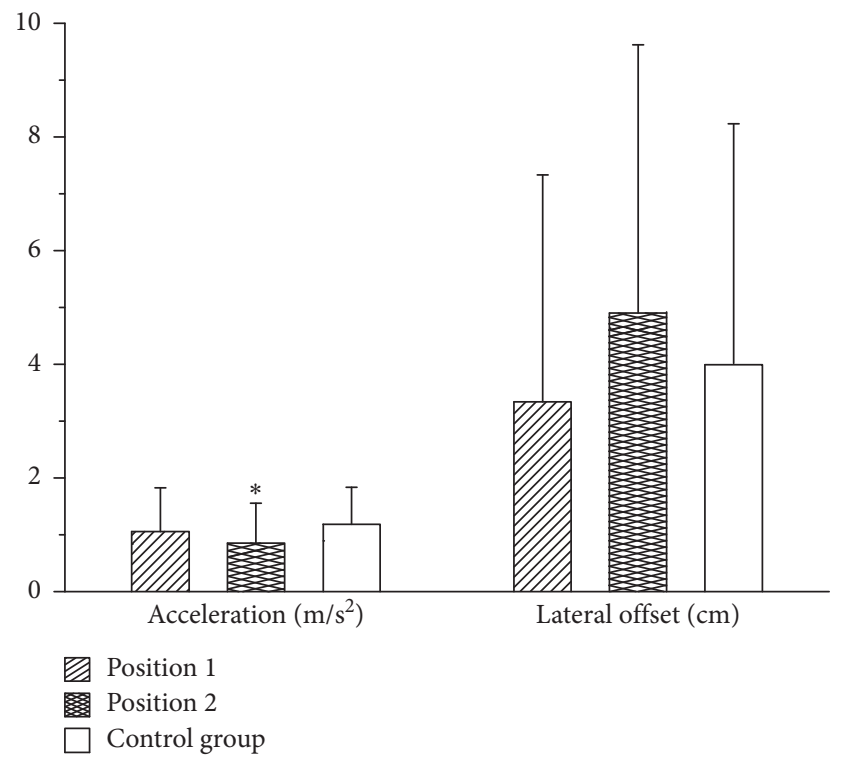

(a)

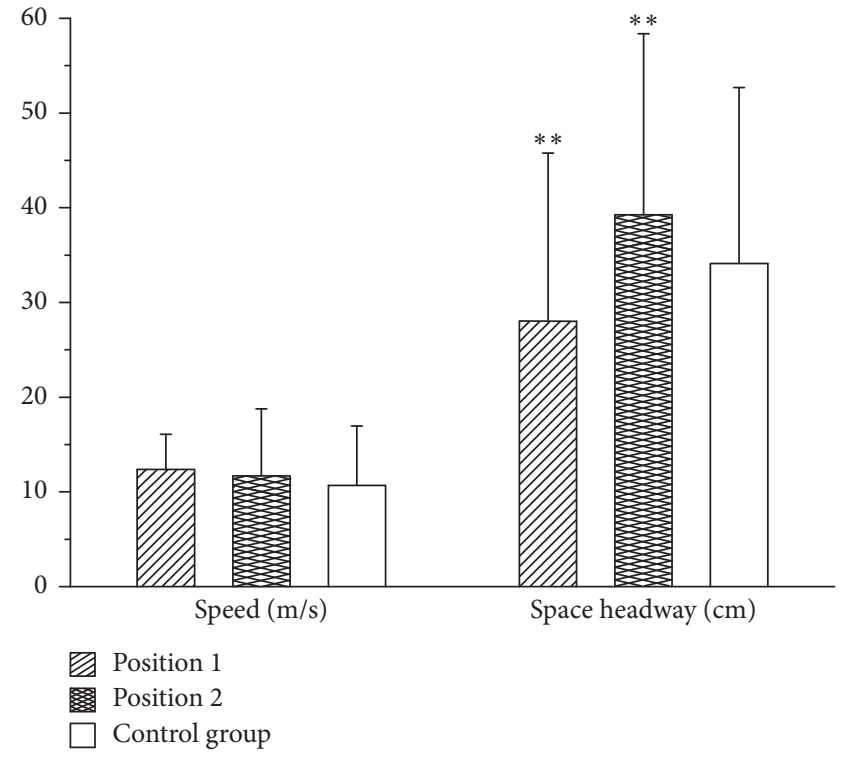

(b)

FIgURE 6: Vehicle indicators for different smartphone positions. (a) Vehicle indicators (part 1); (b) vehicle indicators (part 2).

navigation. There are no significant differences in the blink frequency among the three groups. The blink time of position $2(M=263.78, \mathrm{SD}=6.12)$ is $73.26 \mathrm{~ms}$ higher than that of the control group $(M=190.52, \mathrm{SD}=7.05)$, and the blink time of position $1(M=232.89, \mathrm{SD}=6.88)$ is $42.37 \mathrm{~ms}$ higher than that of the control group. Both position $1(t[138]=$ $1.776, p=0.032)$ and position $2(t[138]=1.966, p=0.005)$ have a significant effect on blink time, especially position 2 . Therefore, the interference of the smartphone on the driver's eye movement indicators at position 2 is greater that than at position 1 .

The three groups of experiments have slight differences in vehicle speed. The order of variance size is as follows: position $2(M=56.55, \mathrm{SD}=5.52)>$ control group $(M=40.07$,
$\mathrm{SD}=5.11)>$ position $1(M=24.80, \mathrm{SD}=4.75)$. It can be seen that the velocity variability of position 2 is larger, and the car has more acceleration and deceleration, whereas the velocity of position 1 remains relatively stable. The acceleration of the control group $(M=1.21, \mathrm{SD}=0.77)$ is $1.21 \mathrm{~m} / \mathrm{s}^{2}$; the acceleration in position $2(M=0.95, \mathrm{SD}=0.88)$ is $0.26 \mathrm{~m} / \mathrm{s}^{2}$ slower, and in position $1(M=1.04, \mathrm{SD}=0.86)$, it is only $0.17 \mathrm{~m} / \mathrm{s}^{2}$ slower. The results of the significance test show that position 2 $(t[138]=1.972, p=0.001)$ has significant effects, whereas position 1 does not, which means that when the phone is in position 2, it has a higher impact on driving speed than position 1. The space headway of position $2(M=39.03$, $\mathrm{SD}=18.62)$ is $10.92 \mathrm{~cm}$ more than position $1(M=28.11$, $\mathrm{SD}=17.25)$, and the control group $(M=34.06, \mathrm{SD}=16.37)$ is 
$5.95 \mathrm{~cm}$ more than position 1 . The variance of position 2 is the largest and the variation of the spacing is the most complicated. Position $1(t[138]=1.955, p=0.001)$ and position $2(t$ $[138]=1.972, p=0.001)$ both had a significant effect on driving. The lateral offset of position $2(M=4.81, \mathrm{SD}=4.02)$ is greater than position $1(M=3.29, \mathrm{SD}=3.71)$ and the control group $(M=3.93 \mathrm{~cm}, \mathrm{SD}=4.21)$, which means that when the smartphone is placed at position 1 , the lateral change of the vehicle is small and relatively safe. Therefore, in terms of vehicle indicators, placement of the smartphone at position 2 had a greater effect on the driver than that at position 1 .

\subsection{The Influence of Different Smartphone Navigation Modes.} The evaluation indicators and significance $(p=0.05)$ of different smart navigation modes are shown in Figures 7 and 8.

The inside fixation frequency of the audio group $(M=5.63, \mathrm{SD}=2.01)$ is lower than that of the control group $(M=20.01, \mathrm{SD}=2.23)$, whereas the inside fixation frequency of the visual group $(M=32.86, \mathrm{SD}=2.13)$ and the audiovisual group $(M=24.86, \mathrm{SD}=2.57)$ were higher than that the control group. The audio group has the highest outside fixation frequency $(M=93.12, \mathrm{SD}=6.22)$ because the largest proportion of drivers paid attention to the road ahead. The order of the average inside fixation time is as follows: audio group $(M=177.52, \mathrm{SD}=12.85)<$ control group $(M=207.32, \quad \mathrm{SD}=11.21)<$ audiovisual group $(M=221.65, \quad \mathrm{SD}=13.65)<$ visual group $\quad(M=251.22$, $\mathrm{SD}=12.66)$. There is little difference in the four groups of experimental data with respect to the average outside fixation time, but the visual group was the smallest $(M=283.6, \mathrm{SD}=22.3 \mathrm{~ms})$. When the driver watches the navigation, the impact on the fixation indicators is greater and the attention on the road outside the vehicle is reduced. Therefore, the effect of the fixation indicators on driving is as follows: visual group $>$ audiovisual group $>$ audio group. The glance range is similar in the three modes, and the effects are not significant. The glance speed of the audio group $(M=141.22, \mathrm{SD}=5.62)$ is $17.36^{\circ}$ faster than that of the control group $(M=123.86, \mathrm{SD}=6.25)$ and the effect is significant $(t[138]=1.801, p=0.033)$, although the glance speed of the visual group $(M=103.65, \mathrm{SD}=5.56)$ and the audiovisual group $(M=117.28, \mathrm{SD}=4.96)$ are slower than that of the control group. It can be seen that when drivers use sound navigation, they can get information quickly in a glance. There is little difference in the blink frequencies of the three navigation modes. The blink time of the modes are all higher than that of the control group $(M=208.79$, $\mathrm{SD}=9.82)$, and the audio group $(M=221.31, \mathrm{SD}=8.11)$ is the closest to the control group. Therefore, the audio group shows the least disturbance to the driver, and the audiovisual group shows the most.

The speeds of the four groups are similar, and the difference between the audio group $(M=12.21, \mathrm{SD}=3.82)$ and the control group $(M=12.42, \mathrm{SD}=4.65)$ is only $0.21 \mathrm{~m} / \mathrm{s}$. The speed variance in the three navigation modes is less than the control group $(M=21.62, \mathrm{SD}=5.67)$, among which the audio group is the smallest $(M=15.05, \mathrm{SD}=3.25)$ and the speed remains stable. The acceleration values of the four groups are also similar. The audio group $(M=0.74, \mathrm{SD}=0.68)$ is the closest to the control group $(M=0.82, \mathrm{SD}=0.61)$, and the visual group $(t[138]=1.942, p=0.001)$ and the audiovisual group $(t[138]=1.975, p=0.001)$ show significant effects. Therefore, the audio group model is more conducive to driving in terms of speed and acceleration. The control group $(M=26.37, \mathrm{SD}=16.25)$ has the smallest space headway, and the audio group $(M=28.66, \mathrm{SD}=15.69 \mathrm{~cm})$ is the closest to it. The variance results are ranked as follows: audio group $(M=246.18, \quad \mathrm{SD}=19.68)<$ control group $(M=257.60$, $\mathrm{SD}=17.96)<$ audiovisual group $(M=284.93, \quad \mathrm{SD}=19.27)$ $<$ visual group $(M=324.36, \mathrm{SD}=22.35)$. This means that the space headway of the audio group showed little change. The lateral offset data of each group shows a small difference, with the maximum difference $0.89 \mathrm{~cm}$. Therefore, the audio group is closer to real driving and showed less interference in terms of vehicle control indicators.

\section{Discussion}

Experiments show that different mobile phone positions and modes have different effects on the driving process. Compared with position 1, position 2 is farther away from the fixation area of the road ahead, and the driver's head moves in a wider range, which increases the glance range and time, so the risk of collision is increased. In addition, the driver's lack of road information can lead to vehicle instability. The driver does not need to transfer outside fixation resources to the inside when using only voice navigation, so the fixation frequency and fixation time of the audio group were significantly lower than that in the visual and audiovisual groups. Because image navigation creates more distraction in the driver's attention, vehicle acceleration has a significant effect on the visual $(t[138]=1.969, p=0.002)$ and audiovisual $(t[138]=1.957, p=0.009)$ groups. This means that the speed varies significantly and can easily cause unsafe driving behavior. The lateral offset of the audio group was the smallest, indicating that the state of the vehicle remains relatively stable while driving.

A study of in-vehicle navigation based on simulated driving showed that a convenient display position with a small visual angle can provide a shorter glance time, but has a higher glance frequency [19]. Compared with position 2, position 1 is closer to the front road area, the glance range used by the driver is smaller, and the fixation time is shorter. This shows that real driving and simulated driving are consistent on this point. However, the fixation frequency at which drivers look inside the vehicle at position 1 is less than position 2, which is inconsistent with the simulated driving conclusions. Because the performance of smartphone navigation is better than in-vehicle navigation, the driver can obtain much comprehensive information via sound at one time and thus the driver does not need to watch the navigation to continue driving. Another simulated driving study showed that in-vehicle navigation above the dashboard seems to be the best [27]. However, in actual driving, if the smartphone is placed above the dashboard as a navigation tool, it will have an impact on the driver's vision, and sudden phone calls and information will greatly interfere with 


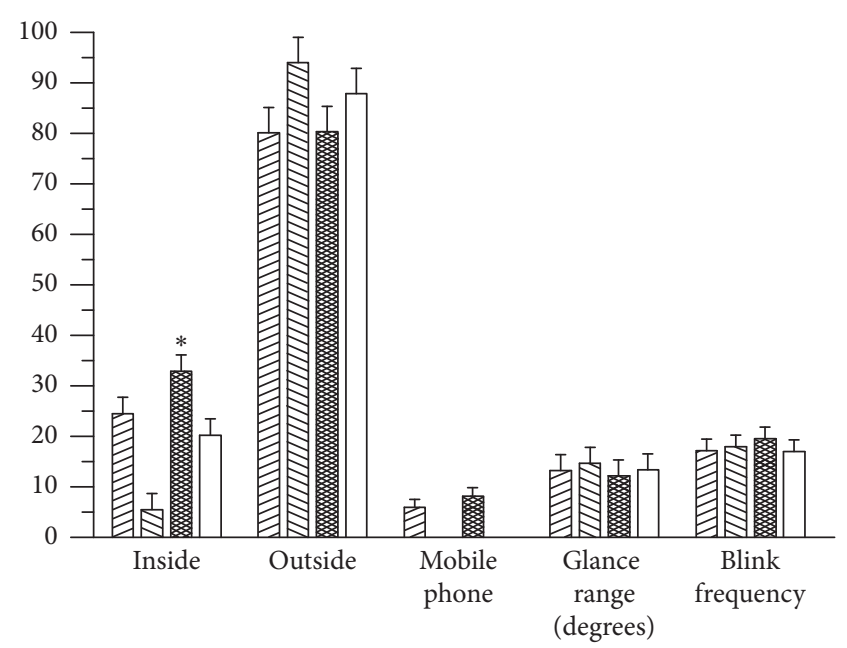

Fixation frequency (three parts)

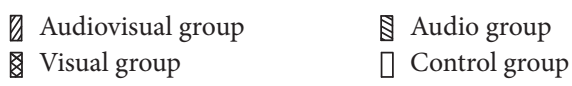

(a)

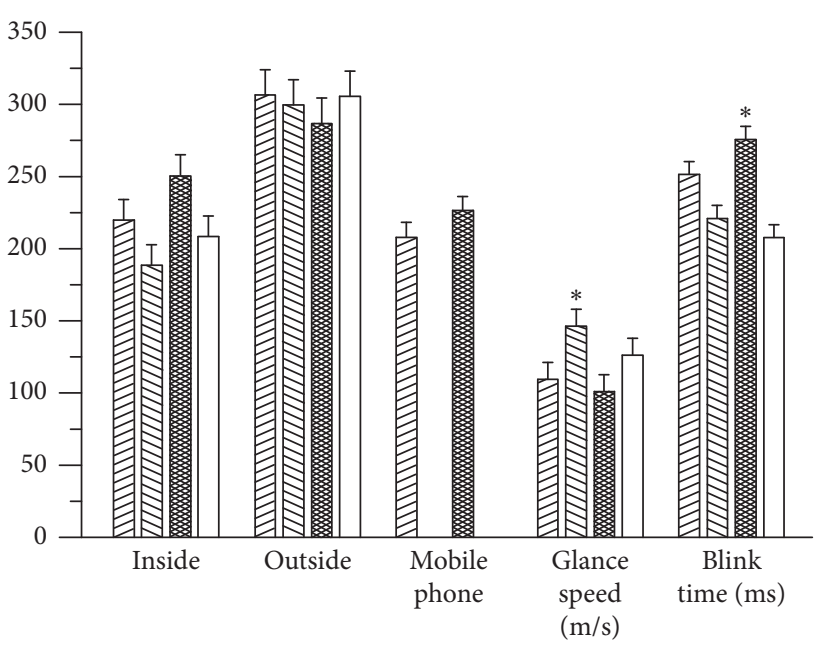

Average fixation time (ms) (three parts)

Audiovisual group Visual group
Audio group

Control group

(b)

Figure 7: Eye movements for different smartphone navigation modes. (a) Eye movements (part 1); (b) eye movements (part 2).

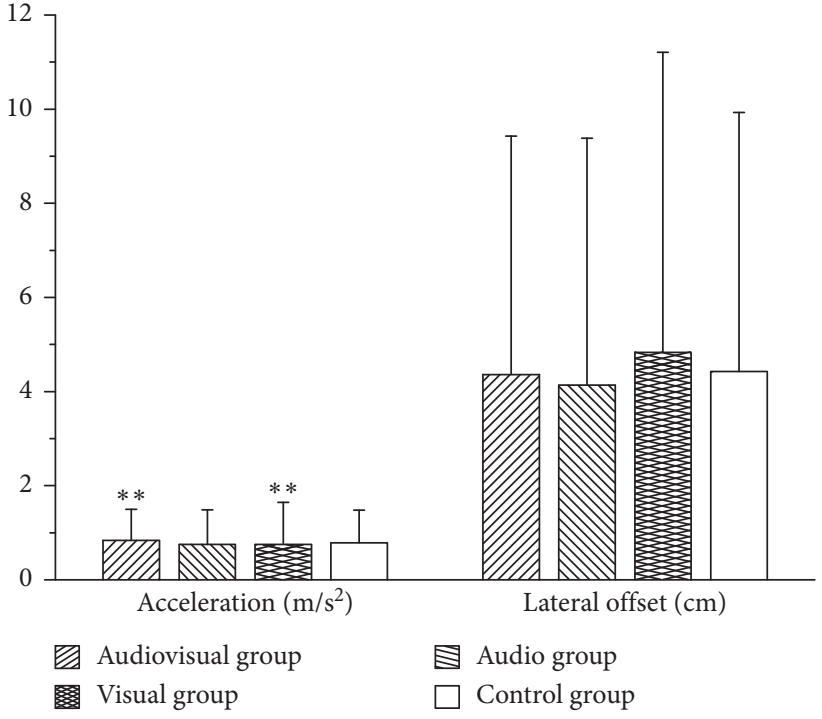

(a)

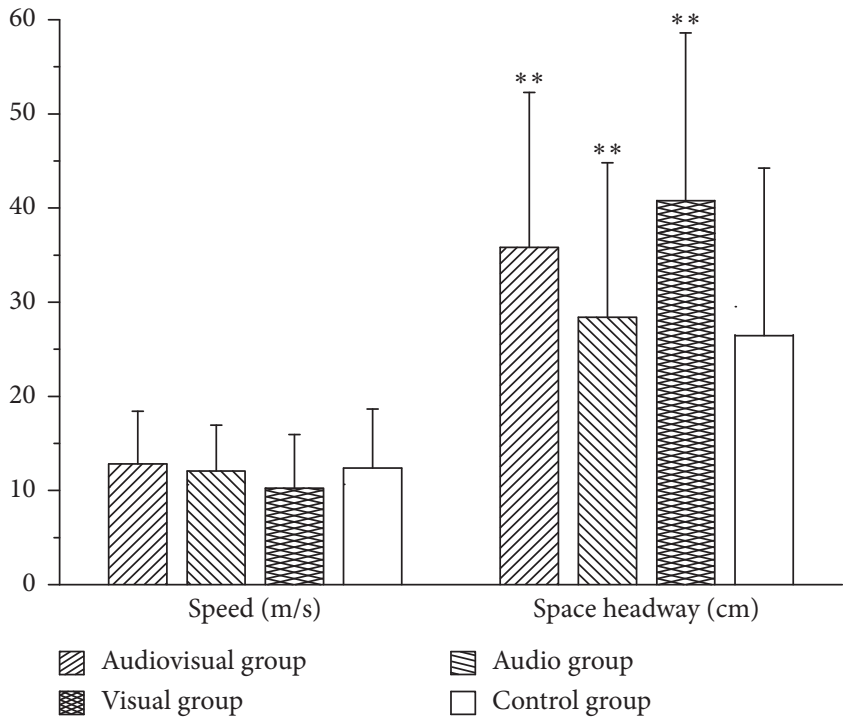

(b)

FiguRE 8: Vehicle indicators for different smartphone navigation modes. (a) Vehicle indicators (part 1); (b) vehicle indicators (part 2).

driving. In addition, lane departure time was used as an indicator of vehicle control, which cannot accurately reflect the impact of navigation on test vehicles. In this paper, the vehicle control indicators are more comprehensive and intuitive, such as lateral offset, acceleration, and speed.

Many researchers have shown that the use of navigation negatively affects the driver. However, navigation still makes sense when considering a driver in unfamiliar circumstances or in need of directions. The conclusion of the study is meaningful for exploring measures to reduce the effects of navigation on driving. It is unsafe for the driver's vision to leave the road ahead for $2 \mathrm{~s}$ while driving [28], but the driver often spends more than $2 \mathrm{~s}$ setting the search for driving routes. When using smartphone navigation, speed changes quickly and lateral offset will occur. Therefore, the driver should try to set driving routes before the vehicle starts. When the navigation information cannot be judged accurately, the speed should be reduced and the vehicle should be stopped if necessary. In addition, from the perspective of software development for smartphones, voice recognition technology should be strengthened to improve the accuracy of human-computer communication and the user friendliness of the interface, so as to reduce the dispersion of drivers' visual resources. 
This paper mainly studied eye movements and vehicle control indicators based on the left cockpit, whereas the cockpit in some countries is located on the right. Whether the difference in driving habits will have an impact on the research conclusions is an interesting topic worthy of further study.

\section{Conclusion}

This paper explores the influence of smartphone navigation on driving behavior based on real driving. The influence of two positions and three navigation modes was analyzed using eye movements and vehicle control indicators. The results show that position 1 is better than position 2 , and the driver is less disturbed by the smartphone. Also, the auditory group experienced the least influence on driving, indicating that the driver can pay more attention to the road when using only voice navigation and maintain better control of the vehicle.

The limitations of this study are that only two mobile phone positions were considered. However, there are some phenomena such as the phone placed on the driver's dashboard, on the left hand side of the vent and at the drink holder. Therefore, we could add more positions to expand this research in the future.

\section{Data Availability}

The data of eye movements and vehicle control used to support the findings of this study are available from the corresponding author upon request.

\section{Conflicts of Interest}

The authors declare that there are no conflicts of interest regarding the publication of this paper.

\section{Acknowledgments}

This work was supported by the Fundamental Research Funds for the Central Universities of China (nos. 300102218410, 300102218521, and 300102218409). The authors thank Lin $\mathrm{Hu}$ for his contributions to this paper.

\section{References}

[1] P. J. Feenstra, J. H. Hogema, and T. Vonk, "Traffic safety effects of navigation systems," in Proceedings of the Intelligent Vehicles Symposium IEEE, pp. 1203-1208, Istanbul, Turkey, June 2007.

[2] Japan Automobile Manufacturers Association, Guideline for In-Vehicle Display Systems (Version 3), Japan Automobile Manufacturers Association, Tokyo, Japan, 2004.

[3] Standardization Administration of the People's Republic of China (GB/T 19392-2003), General Specification for In-Vehicle GPS Navigation System, Standardization Administration of the People's Republic of China, Beijing, China, 2003.

[4] W.-C. Lee, M.-C. Ma, and B.-W. Cheng, "Field comparison of driving performance using a portable navigation system," Journal of Navigation, vol. 63, no. 1, pp. 39-50, 2010.
[5] W.-C. Lee and B.-W. Cheng, "Comparison of portable and onboard navigation system for the effects in real driving," Safety Science, vol. 48, no. 10, pp. 1421-1426, 2010.

[6] W.-C. Lee and B.-W. Cheng, "Effects of using a portable navigation system and paper map in real driving," Accident Analysis \& Prevention, vol. 40, no. 1, pp. 303-308, 2008.

[7] C. D. Wickens, "Multiple resources and performance prediction," Theoretical Issues in Ergonomics Science, vol. 3, no. 2, pp. 159-177, 2002.

[8] P. A. Hancock, M. Lesch, and L. Simmons, "The distraction effects of phone use during a crucial driving maneuver," Accident Analysis \& Prevention, vol. 35, no. 4, pp. 501-514, 2003.

[9] ISO/DIS 26022, Road Vehicles-Ergonomic Aspects of Transport Information and Control Systems-Simulated Lane Change Test to Assess In-Vehicle Secondary Task Demand, International Organization for Standardization, Geneva, Switzerland, 2007.

[10] A. K. Huemer and M. Vollrath, "Driver secondary tasks in Germany: using interviews to estimate prevalence," Accident Analysis \& Prevention, vol. 43, no. 5, pp. 1703-1712, 2011.

[11] H. Rouzikhah, M. King, and A. Rakotonirainy, "Examining the effects of an eco-driving message on driver distraction," Accident Analysis \& Prevention, vol. 50, no. 2, pp. 975-983, 2013.

[12] K. M. White, M. K. Hyde, S. P. Walsh, and B. Watson, "Mobile phone use while driving: an investigation of the beliefs influencing drivers' hands-free and hand-held mobile phone use," Transportation Research Part F: Traffic Psychology and Behaviour, vol. 13, no. 1, pp. 9-20, 2010.

[13] C. J. Lin and H. J. Chen, "Verbal and cognitive distractors in driving performance while using hands-free phones," Perceptual and Motor Skills, vol. 103, no. 3, pp. 803-810, 2006.

[14] W. Brodsky, "The effects of music tempo on simulated driving performance and vehicular control," Transportation Research Part F: Traffic Psychology and Behaviour, vol. 4, no. 4, pp. 219-241, 2001.

[15] R. Dewar, "In-vehicle information and driver overload," International Journal of Vehicle Design, vol. 9, pp. 557-564, 1998.

[16] M. Sodhi, B. Reimer, and I. Llamazares, "Glance analysis of driver eye movements to evaluate distraction," Behavior Research Methods, Instruments, \& Computers, vol. 34, no. 4, pp. 529-538, 2002.

[17] M. Sodhi, B. Reimer, J. L. Cohen et al., "On-road driver eye movement tracking using head-mounted device," in Proceedings of the Louisiana Eye Tracking Research \& Applications Symposium, pp. 61-68, New Orleans, LA, USA, March 2002.

[18] B. Metz, N. Schömig, and H.-P. Krüger, "Attention during visual secondary tasks in driving: adaptation to the demands of the driving task," Transportation Research Part F: Traffic Psychology and Behaviour, vol. 14, no. 5, pp. 369-380, 2011.

[19] R. Zheng, K. Nakano, H. Ishiko, K. Hagita, M. Kihira, and T. Yokozeki, "Eye-gaze tracking analysis of driver behavior while interacting with navigation systems in an urban area," IEEE Transactions on Human-Machine Systems, vol. 46, no. 4, pp. 546-556, 2016.

[20] D. B. Kaber, Y. Liang, Y. Zhang, M. L. Rogers, and S. Gangakhedkar, "Driver performance effects of simultaneous visual and cognitive distraction and adaptation behavior," Transportation Research Part F: Traffic Psychology and Behaviour, vol. 15, no. 5, pp. 491-501, 2012.

[21] Y.-C. Liu, "Comparative study of the effects of auditory, visual and multimodality displays on drivers' performance in 
advanced traveller information systems," Ergonomics, vol. 44, no. 4, pp. 425-442, 2001.

[22] K. A. Brookhuis, C. J. G. van Driel, T. Hof, B. van Arem, and M. Hoedemaeker, "Driving with a congestion assistant; mental workload and acceptance," Applied Ergonomics, vol. 40, no. 6, pp. 1019-1025, 2009.

[23] E. Cheung, A. Bera, E. Kubin et al., "Identifying driver behaviors using trajectory features for vehicle navigation," in Proceedings of the IEEE/RSJ International conference on Intelligent Robots and Systems, Madrid, Spain, October 2018.

[24] E. Cheung, A. Bera, and D. Manocha, "Efficient and safe vehicle navigation based on driver behavior classification," in Proceedings of the IEEE/CVF Conference on Computer Vision and Pattern Recognition Workshops, IEEE Computer Society, Long Beach, CA, USA, June 2018.

[25] L. Zhao and H. Liu, "Visual characteristics of new and old drivers in the complex environment of rural road," China Safety Science Journal (in Chinese), vol. 26, no. 9, pp. 30-34, 2016.

[26] G. Underwood, D. Crundall, and P. Chapman, "Selective searching while driving: the role of experience in hazard detection and general surveillance," Ergonomics, vol. 45, no. 1, pp. 1-12, 2002.

[27] M. Wittmann, M. Kiss, P. Gugg et al., "Effects of display position of a visual in-vehicle task on simulated driving," Applied Ergonomics, vol. 37, no. 2, pp. 187-199, 2006.

[28] B. G. Simons-Morton, F. Guo, S. G. Klauer, J. P. Ehsani, and A. K. Pradhan, "Keep your eyes on the road: young driver crash risk increases according to duration of distraction," Journal of Adolescent Health, vol. 54, no. 5, pp. 61-67, 2014. 


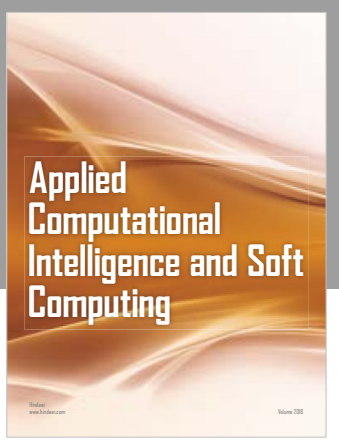

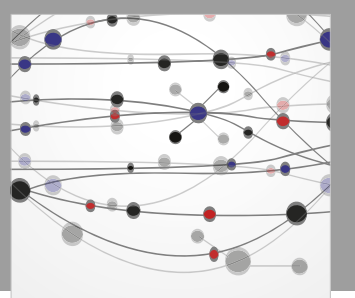

The Scientific World Journal
Submit your manuscripts at

Computing
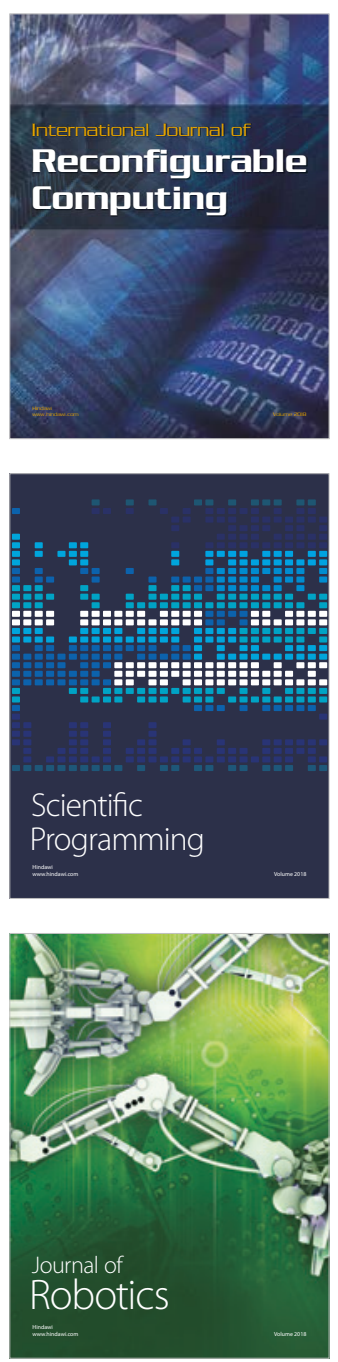

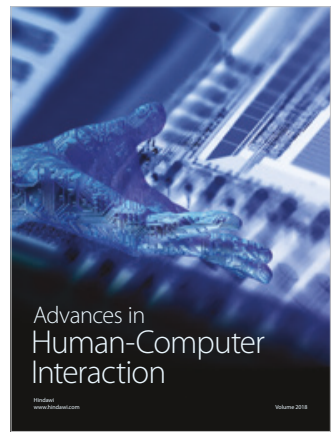

Human-Compute

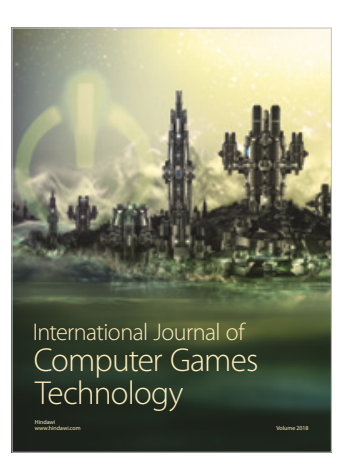

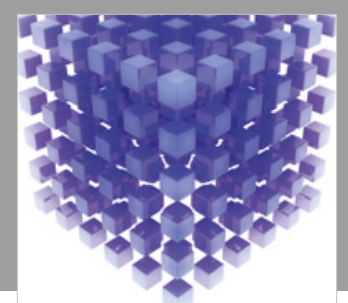

Mathematical Problems in Engineering

\section{Engincering}
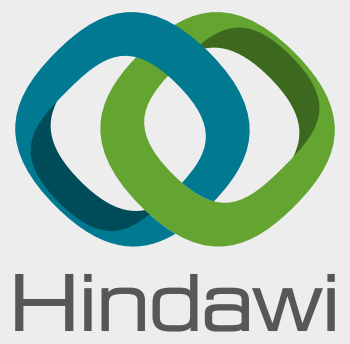

www.hindawi.com
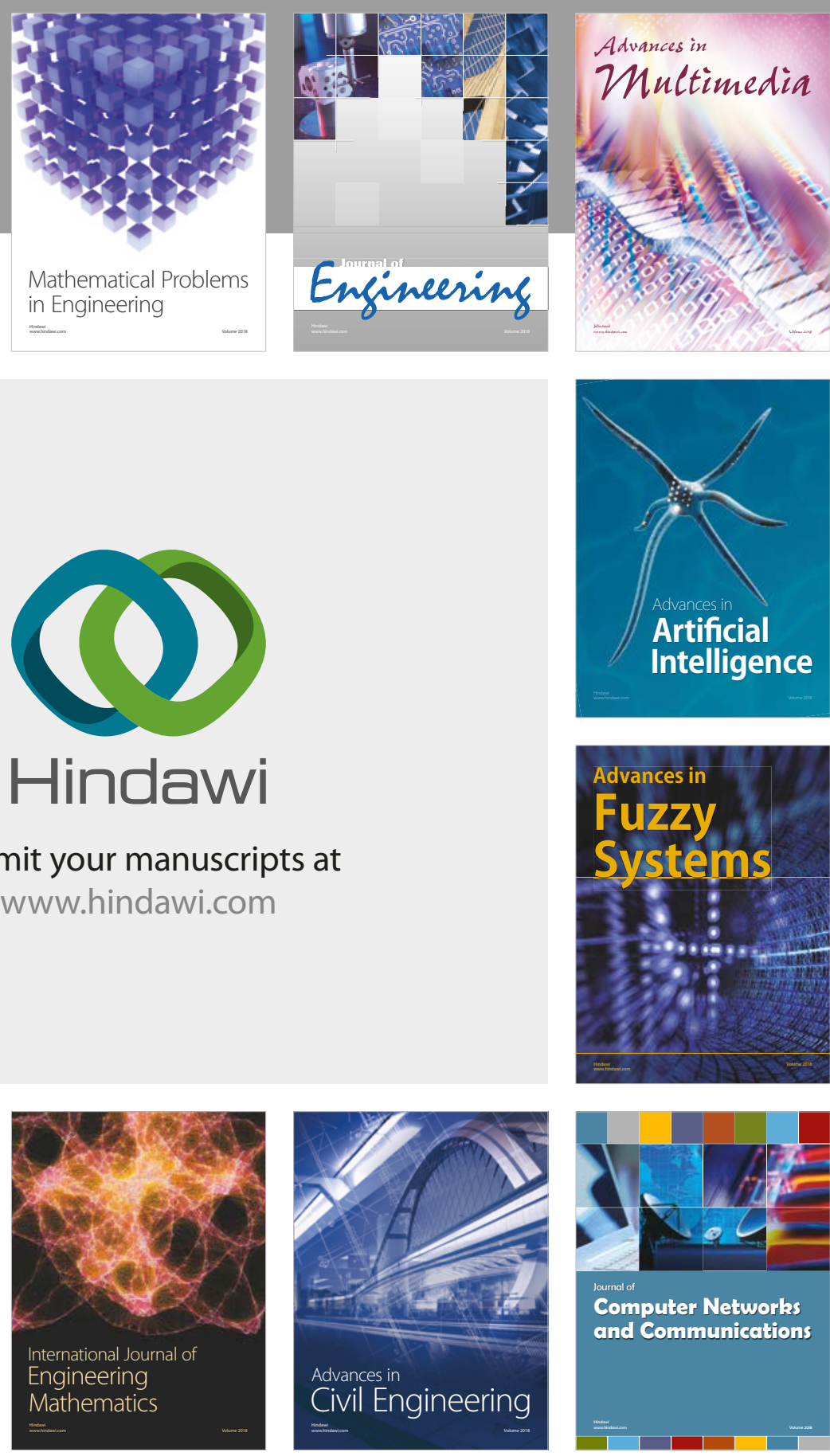

Computer Networks and Communications

Multimedia
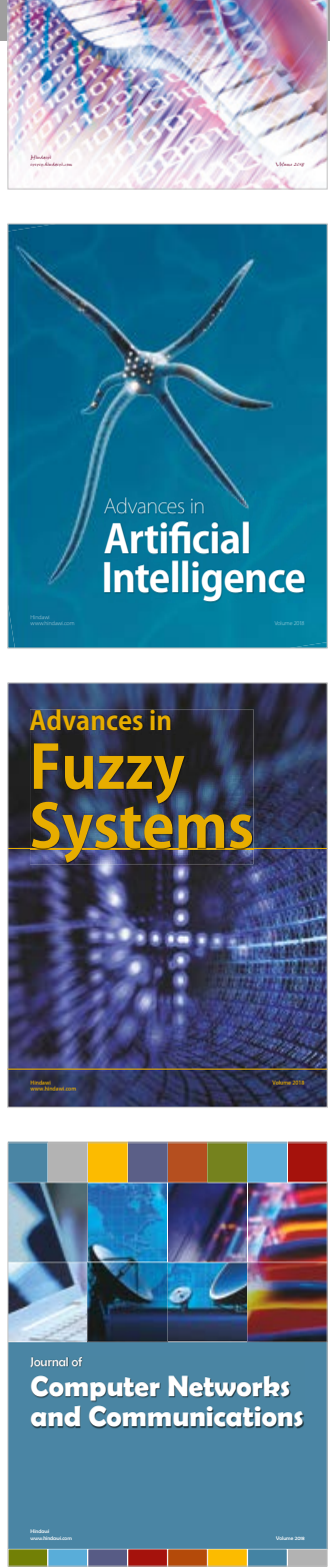

Advances in

Modelling \&

Simulation

in Engineering

interaction

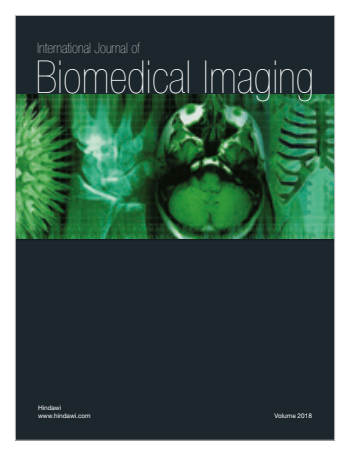

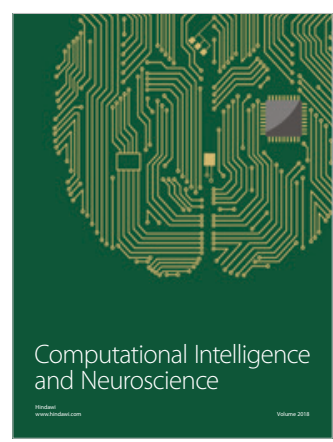

